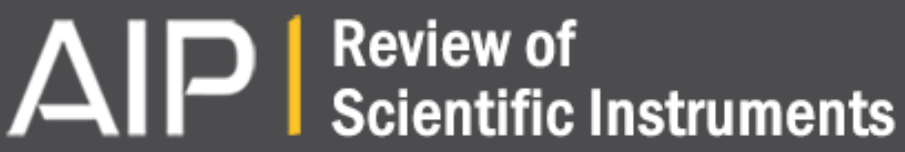

\section{Development of the imaging system of the balloon-borne gamma-ray telescope Máscara Codificada (MASCO)}

João Braga, Flavio D’Amico, Thyrso Villela, Jorge Meja, Raphael A. Fonseca, and Elisete Rinke

Citation: Review of Scientific Instruments 73, 3619 (2002); doi: 10.1063/1.1505841

View online: http://dx.doi.org/10.1063/1.1505841

View Table of Contents: http://scitation.aip.org/content/aip/journal/rsi/73/10?ver=pdfcov

Published by the AIP Publishing

\section{Articles you may be interested in}

Polarimetric performance of a Laue lens gamma-ray CdZnTe focal plane prototype

J. Appl. Phys. 104, 084903 (2008); 10.1063/1.3000092

A fast star sensor for balloon payloads

Rev. Sci. Instrum. 74, 4169 (2003); 10.1063/1.1602961

Attitude control system for balloon-borne experiments

AIP Conf. Proc. 616, 56 (2002); 10.1063/1.1475603

A new telemetry system for a balloon-borne far-infrared astronomical telescope

Rev. Sci. Instrum. 71, 281 (2000); 10.1063/1.1150194

Coded-aperture $\mathrm{x}$ - or -ray telescope with least-squares image reconstruction. III. Data acquisition and analysis enhancements

Rev. Sci. Instrum. 68, 2404 (1997); 10.1063/1.1148124

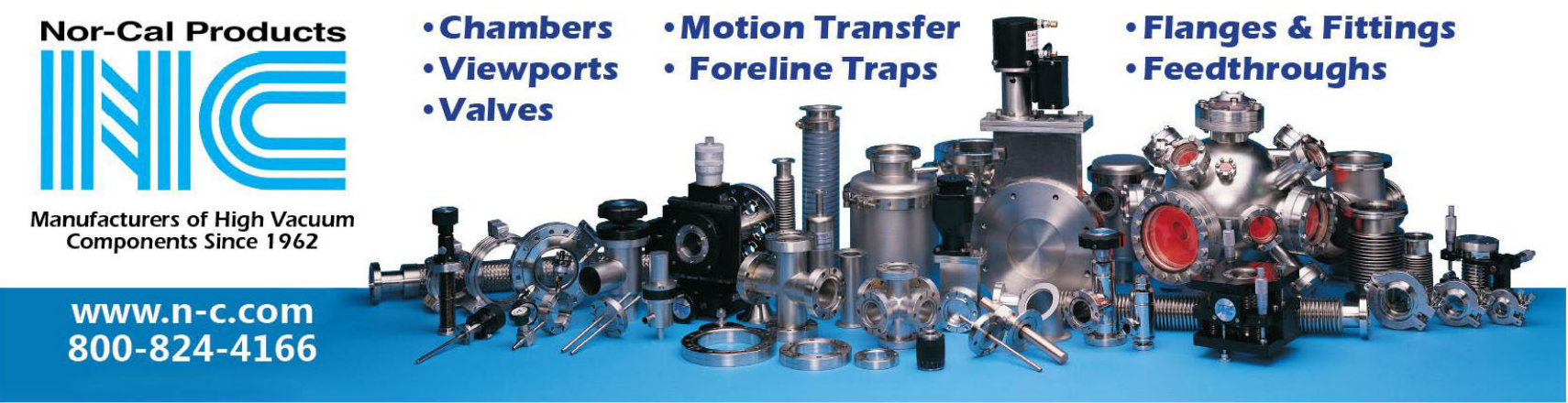




\title{
Development of the imaging system of the balloon-borne gamma-ray telescope Máscara Codificada (MASCO)
}

\author{
João Braga, ${ }^{\text {a) }}$ Flavio D’Amico, Thyrso Villela, Jorge Mejía, Raphael A. Fonseca, \\ and Elisete Rinke \\ Instituto Nacional de Pesquisas Espaciais (INPE), Divisão de Astrofísica, C.P. 515, São José dos Campos, \\ SP, CEP 12201-970, Brazil
}

(Received 21 March 2002; accepted for publication 28 June 2002)

\begin{abstract}
We report laboratory imaging results of a balloon-borne gamma-ray imaging telescope, Máscara Codificada (Portuguese for "coded mask,") designed to obtain high angular resolution $(\sim 14$ arcmin) images of the sky in the $50 \mathrm{keV}-1.8 \mathrm{MeV}$ energy range. The instrument incorporates a coded mask with an aperture pattern based on a $19 \times 19$-element modified uniformly redundant array (MURA). This pattern belongs to a subclass of MURAs that are almost completely antisymmetrical for $90^{\circ}$ rotations with respect to the lower-right corner of the central element, which allows the implementation of an antimask by a single rotation of the whole mask by $90^{\circ}$. The symmetry properties of the MURAs are discussed. The algorithm for determining the position of the gamma-ray interactions on the instrument's main detector is described and the results of laboratory tests of the imaging system are presented. The mask-antimask subtraction, applied after a flat-fielding procedure, produced a $60 \%$ increase in the signal-to-noise ratio of a strong $(\sim 100 \sigma)$ point source (662 $\mathrm{keV}$ photons coming from a ${ }^{137} \mathrm{Cs}$ radioactive source) image by eliminating systematic distortions in the instrumental background measured over the detector plane. (c) 2002 American Institute of Physics. [DOI: 10.1063/1.1505841]
\end{abstract}

\section{INTRODUCTION}

The coded-aperture imaging concept ${ }^{1,2}$ has been used with great success over the last decade to produce images of the sky at hard x-ray and low-energy gamma-ray energies. The space instruments Spacelab-2/XRT, ${ }^{3}$ GRANAT/ART-P, ${ }^{4}$ BeppoSAX/WFC, ${ }^{5}$ and RXTE/ASM, ${ }^{6}$ as well as balloonborne telescopes GRIP $^{7}$ and EXITE $^{8,9}$ have all successfully employed coded-aperture techniques above a few $\mathrm{keV}$. In the low-energy gamma-ray range, the SIGMA ${ }^{10-12}$ telescope aboard the GRANAT spacecraft was particularly successful and obtained remarkable gamma-ray images of several regions of the sky, especially the central region of our galaxy. ${ }^{13-16}$

The basic idea of the coded-aperture imaging technique with stationary masks is to spatially encode the incoming high-energy photons in such a way that each sky resolution element (a "sky bin") in the telescope's field of view (FOV) produces a unique pattern of counts over a position-sensitive detector (PSD). This is achieved by placing an opaque plate with an especially designed pattern of openings (the coded mask) in the FOV at a suitable distance from the detector so as to achieve the desired combination of angular resolution and FOV. The sensitivity of such an instrument depends not only on the effective area of the detector and on the instrumental background, as is usual for conventional $x$ - and $\gamma$-ray telescopes, but also on properties of the mask (open fraction and intrinsic noise) and on the degree of blurring of the count

\footnotetext{
${ }^{\text {a)} E l e c t r o n i c ~ m a i l: ~ b r a g a @ d a s . i n p e . b r ~}$
}

distribution on the PSD, which is governed by the detector spatial resolution.

The detector count distribution can be mathematically described as a cross correlation between the source map in the sky and the pattern of openings in the mask. The image is then reconstructed essentially by a correlation between the count distribution in the PSD and a suitably chosen decoding function $G$, which in general mimics the mask pattern. A major breakthrough in coded-aperture imaging occurred when Fenimore and Cannon ${ }^{17}$ introduced a family of arrays (the uniformly redundant arrays - URAs) which, when used as the pattern of openings in a coded mask, allows imaging with no inherent noise and maximum sensitivity (in a URA mask, approximately half of the area is open). Since the autocorrelation of a URA is a delta function, the $G$ function for the URAs is a unimodular representation of the mask pattern. Later, Gottesman and Fenimore ${ }^{18}$ noticed that a slight modification in the $G$ function permits the use of a new class of arrays, the "modified uniformly redundant arrays" (MURAs), which are built using the same algorithm and have the same imaging properties of the URAs, with the additional advantage of being square (see Sec. III). The use of the MURAs increased the number of available square or nearly square mask patterns (with unimodular $G$ functions and $\sim 50 \%$ throughput) by a factor of $\sim 3$, thus providing a wider selection of mask patterns to suit particular experimental constraints.

In this article we report laboratory imaging results produced by the Máscara Codificada (MASCO) $\gamma$-ray telescope, which utilizes a coded mask based on a $19 \times 19$ MURA pattern. In Sec. II the experiment is briefly described, with emphasis on the imaging system. In Sec. III we point out important symmetry properties of a subclass of MURA patterns 


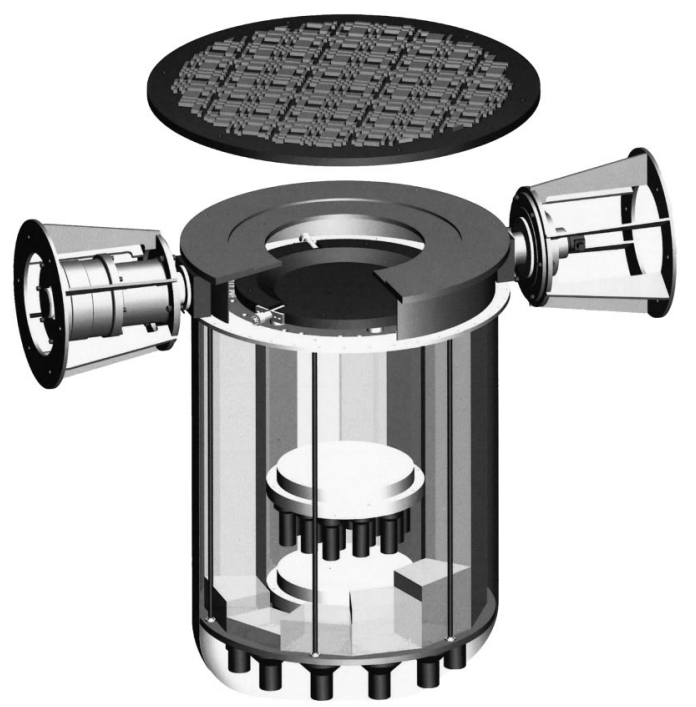

FIG. 1. A general view of the imaging system of the MASCO experiment with the distance to the mask not to scale. The detector system is placed inside a pressure vessel and the distance from the mask to the detector surface is $3.05 \mathrm{~m}$.

that allow the implementation of an antimask by a simple $90^{\circ}$ rotation of the mask structure. In Sec. IV we describe the algorithm used to determine the positions and energies of $\gamma$-ray interactions in the PSD. In Sec. V, the results of laboratory tests of the imaging system of the telescope are discussed. Finally, in Sec. VI we discuss the sensitivity of the experiment and show a simulated image of the Galactic Center (GC) region.

\section{DESCRIPTION OF THE INSTRUMENT}

The MASCO experiment ${ }^{19,20}$ is a balloon-borne imaging gamma-ray telescope designed to operate in the $50 \mathrm{keV}-1.8$ $\mathrm{MeV}$ energy range with a $\sim 14$ arcmin angular resolution. The telescope has a circular $13.6^{\circ}$-diam fully coded field of view (FCFOV) surrounded by a partially coded field of view (PCFOV) up to $23.5^{\circ}$. A sketch of the imaging system of the telescope is shown in Fig. 1. The detector system is kept at $\sim 1$ atm pressure inside a vessel to avoid corona discharges within the high voltage power supplies and also to provide a thermally controlled, electrically shielded environment for the enclosed instrumentation at balloon altitudes. The main detector is a 40.6-cm-diam, 5.08-cm-thick NaI(Tl) scintillator (with a $0.76-\mathrm{mm}$-thick $\mathrm{Al}$ entrance window) optically coupled to $197.62-\mathrm{cm}$-diam photomultiplier tubes (PMT) in an Anger Camera configuration. Figure 2 shows the positions of the 19 PMTs with respect to the detector. The detector is surrounded by an active shield of large-volume plastic scintillators at the sides and by an identical $\mathrm{NaI}(\mathrm{Tl})$ detector at the bottom. ${ }^{21}$ A cylindrical top plate of 3-mm-thick plastic scintillator is placed in front of the detector for charged particle veto. The three PMTs of the top plate are placed on its side (on special mounts) spaced by $120^{\circ}$. The MASCO detector system is similar to the one in Caltech's GRIP experiment. $^{22}$

The coded mask, placed at a distance of $3.05 \mathrm{~m}$ from the detector surface, is a $1-\mathrm{m}$-diam disk with a cyclic repetition

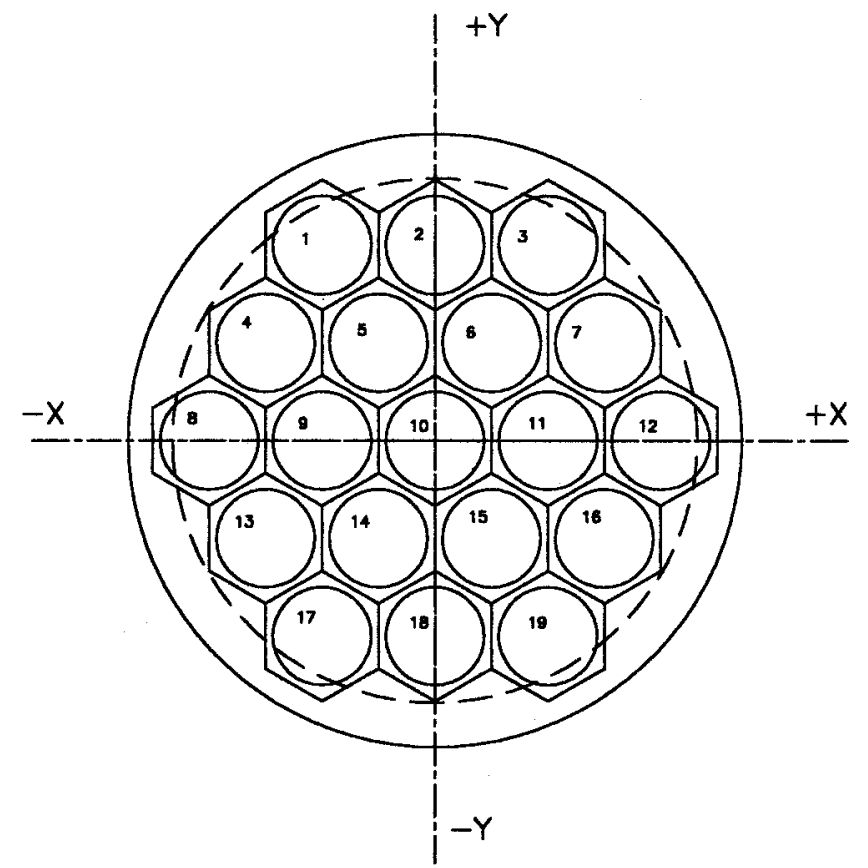

FIG. 2. Geometrical arrangement of the 19 PMTs optically coupled to the main $\mathrm{NaI}(\mathrm{Tl})$ detector. The dashed circle indicates the edge of the actual crystal. The axis provides the reference system for the position determination algorithm.

of a $19 \times 19$ MURA pattern made of $1.25-\mathrm{cm}$-side, 2-cmthick square lead elements. The mask structure is allowed to rotate in order to change the configuration from "mask" to "antimask" and vice versa. Furthermore, a continuous rotation of the mask (at about $1 \mathrm{rpm}$ ) provides a time modulation of the signal that enables the determination of unambiguous source locations. ${ }^{23}$ Due to the rotation of the mask and the sky rotation caused by the diurnal motion during exposures, our data reduction procedure includes the acquisition of short-exposure images for which neither the mask rotation nor the sky rotation produces significant differences in the orientation and displacement of the shadowgram cast in the detector (the position displacements are small fractions of the detector spatial resolution). The photon interaction positions in each short integration are then corrected for the combination of the two effects and then the raw reoriented images are added.

The experiment is placed in a stabilized balloon platform in an alt-azimuthal configuration and the telescope is able to carry out pointed observations with at least 6 arcmin accuracy. The instrument employs two charge coupled device star cameras for field recognition and guidance. During daytime observations, a sun sensor and a sun tracker are used. The payload also employs gyroscopes and magnetometers and is automatically guided. For a description of the gondola and its guiding algorithms and strategies, see Refs. 23, 24, and 25. In Table I, an outline of the experiment is shown.

\section{SYMMETRY PROPERTIES OF THE MURAS}

The MURA patterns are built using essentially the same algorithm of the rectangular URAs, with the exception that they are square arrays and can be built from any prime num- 
TABLE I. An overview of the MASCO balloon-borne coded-aperture gamma-ray telescope.

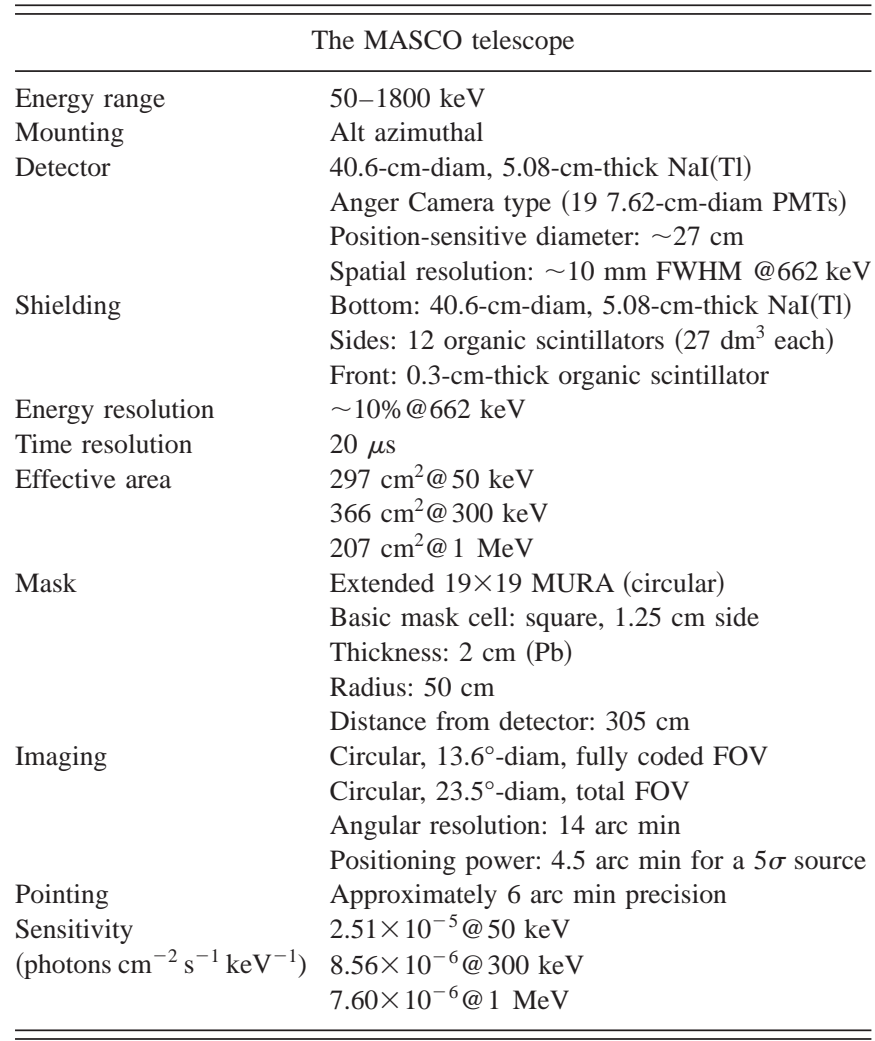

ber (the URAs can only be built with two prime numbers which differ by two). Gottesman and Fenimore ${ }^{18}$ realized that the modification of one particular element in the pattern leads to a decoding function that provides "perfect" imaging properties to the MURAs, in the sense that there is no intrinsic noise inherent in the decoding procedure. This means that if $M$ is the MURA pattern and $G$ is the decoding function, $M * G$ (where $*$ is the correlation operator) is a $\delta$ function. An additional feature is the fact that a subset of the MURAs shows $90^{\circ}$ antisymmetry (except for one element), a fact that was first reported in the literature by Byard ${ }^{26}$ and was discovered independently by us. ${ }^{27}$ This makes this subclass of MURAs suitable for the implementation of antimasks in gamma-ray telescopes by performing a single $90^{\circ}$ rotation of the mask supporting structure (a similar-albeit a little more complicated-way of obtaining an antimask from the original mask for the case of URA masks had been already discovered and implemented by our group in the TIMAX experiment $^{28-31}$ ). Moreover, we noticed that all the remaining MURAs show $90^{\circ}$ partial symmetry: all the elements, except the elements of the opaque first line and the open first column, remain the same under $90^{\circ}$ rotations.

In Fig. 3(a) we show the pattern of the coded-mask used by the MASCO telescope, which is a cyclic repetition of a $19 \times 19$-element MURA. The center of rotation of the mask is the lower-right corner of the central element of the central MURA pattern. In Fig. 3(b) we show the mask rotated by $90^{\circ}$ and in Fig. 3(c) we show the elements that changed from open to closed or vice versa by performing this rotation. The black elements are the only ones that did not change; these elements correspond to $M(1,1)$ in the aperture pattern. As

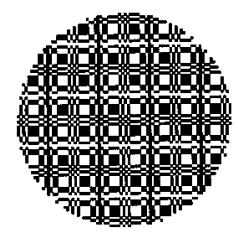

(a)

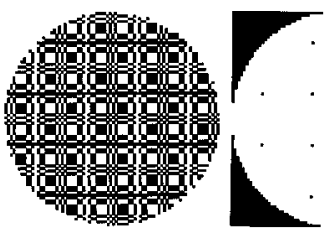

(b)

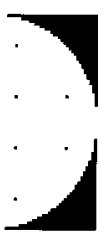

(c)
FIG. 3. (a) The pattern of coded-mask of the MASCO experiment. The basic pattern is a $19 \times 19$ MURA. (b) The same mask rotated by $90^{\circ}$ to show the antisymmetrical property. (c) The black elements are the ones that do not get interchanged by the $90^{\circ}$ rotation; all the white elements changed from closed to open or vice versa.

pointed out by Byard, ${ }^{26}$ if the correlation of the subtracted array (mask-antimask) is performed with a $G$ function that has a value of 0 in the $(1,1)$ position, instead of the usual " -1 ," the reconstructed image is still perfect (no intrinsic noise).

In Fig. 4(a) we show a coded mask based on a $17 \times 17$ MURA pattern. Taking the same procedure as in Fig. 3, we see that a $90^{\circ}$ rotation of the mask produces an identical pattern, except for the first line and first column of the basic array, that get swapped, as shown in Figs. 4(b) and 4(c).

\section{POSITION DETERMINATION ON THE DETECTOR}

In order to determine the $(x, y)$ position of the $\gamma$-ray events in the detector, we utilize a method based on an algorithm developed for a tomography scintillation camera. ${ }^{32} \mathrm{We}$ first define a Cartesian coordinate system on the surface of the detector based on the spatial orientation of the 19 PMTs (see Fig. 2). When an event occurs, each PMT signal, after being preamplified, is fed into four resistors. The values of the resistances are selected in such a way as to compensate for the different PMT locations and for the distances from the PMTs to the center of the detector (the values are roughly inversely proportional to the distance of the PMT to the $X$ and $Y$ axis and depend on which quadrant the PMT is located). In this way, each event in the detector generates electronic signals in four "electronic lines" $(+X,-X,+Y$, and $-Y$ ) which connect the resistors together in a network. The gain of each PMT-preamplifier pair has to be adjusted in order to produce the same pulse height for monoenergetic photons hitting the detector exactly in the center of each PMT (this is achieved by collimating radioactive sources in the laboratory).

The position can then be obtained by

$$
X_{\mathrm{ele}}=\frac{(+X)-(-X)}{(+X)+(-X)}, \quad Y_{\mathrm{ele}}=\frac{(+Y)-(-Y)}{(+Y)+(-Y)} .
$$

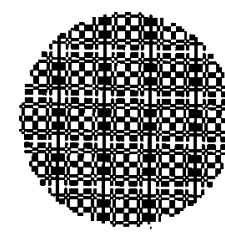

(a)

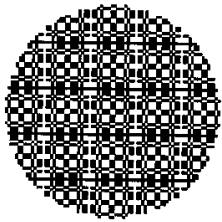

(b)

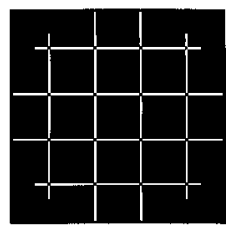

(c)
FIG. 4. Same as in Fig. 3, except that now the basic pattern is a $17 \times 17$ MURA. One can see that this pattern is not antisymmetrical, being actually partially symmetrical with respect to the $90^{\circ}$ rotation. 


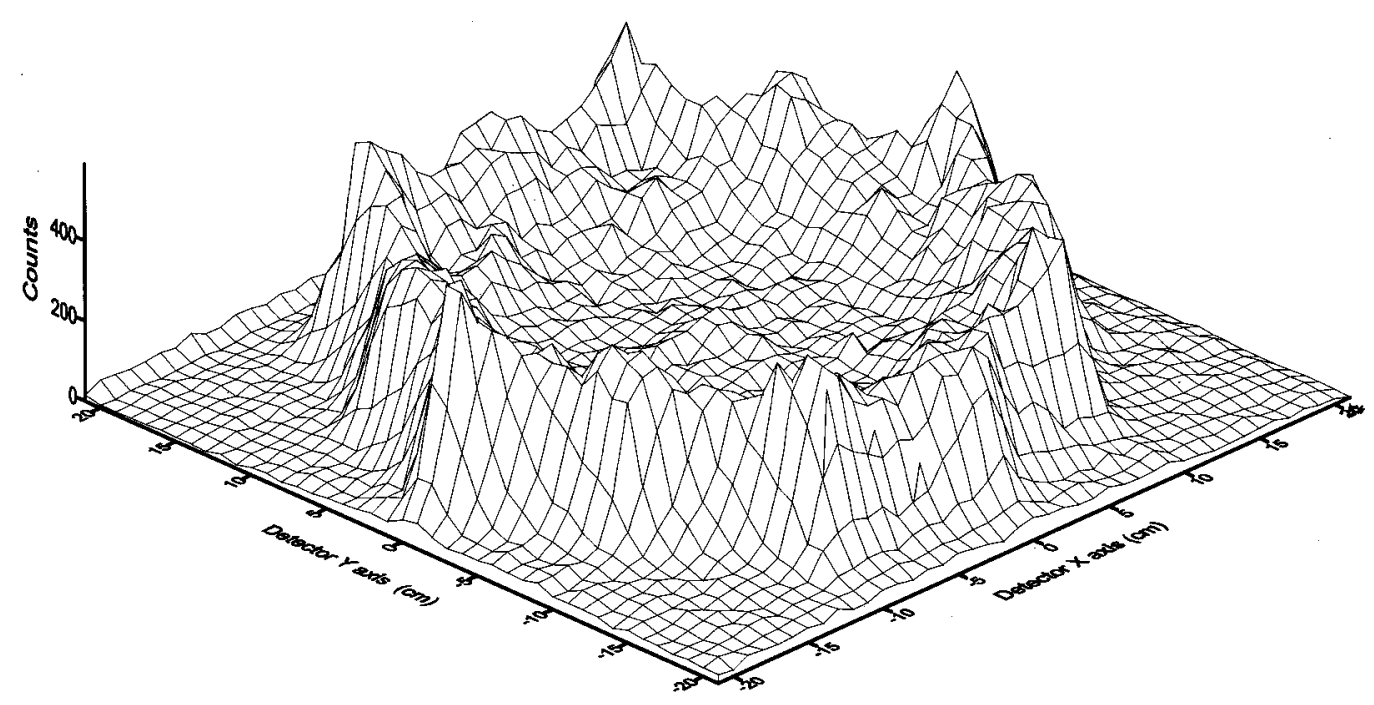

FIG. 5. Uncorrected lab background count distribution over the detector. The two-dimensional histogram samples the surface at 1/4 the mask cell size.

To transform from these electronic coordinates to real geometric coordinates, we simply calculate

$$
X_{\text {geo }}=\kappa X_{\text {ele }}, \quad Y_{\text {geo }}=\kappa Y_{\text {ele }},
$$

where $\kappa$ is a calibration constant.

To obtain the energy of the detected photon, we perform a sum of the 19 PMT signals, since the energy is proportional to the amount of photoelectrons generated in all PMTs. An alternative way to calculate the energy of an event is to add up the signals from the four electronic lines. Energy spectra of a collimated ${ }^{137} \mathrm{Cs}$ (662 keV line) radioactive source taken in the laboratory have shown virtually no variation with position and an energy resolution of $\sim 10 \%$.

\section{LABORATORY IMAGES}

In order to test the performance of the imaging system of the MASCO telescope, we have carried out calibration procedures and produced images of radioactive sources in the laboratory.

In Fig. 5 we show a two-dimensional position histogram of a long ( $\sim 6 \mathrm{~h})$ integration of the background at the laboratory, sampled with a linear scale of $1 / 4$ the size of one mask element. We can clearly see a relative increase in the recorded counts towards the edge of the detector. This can be attributed both to position-dependent background systematics and degradation of the detector ability to measure correct positions at large radii. When gamma rays hit the detector at radii greater than $r \sim 13 \mathrm{~cm}$, the positions cannot be determined unambiguously. The recorded positions cluster around $r \sim 13 \mathrm{~cm}$, with an additional tendency to concentrate around the sites of the external PMTs. This leads to the wrong number of events being attributed to positions at increasing radii, providing an apparent variation in quantum efficiency across the detector. Even though the sensitivity of the detector does not vary significantly across its surface (the light flash produced by a gamma-ray anywhere in the crystal is detected by the PMTs every time), the background count distribution over the detector surface has an additional dependency on quantum efficiency variations among the 19 PMTs, since a low quantum efficiency of one particular PMT would degrade both the energy resolution and the position resolution of the detector as a whole. However, differences in quantum efficiency among the PMTs will not affect the centroid of the measured position distribution of events corresponding to gamma rays hitting the detector surface at a given position, since the output signals of all PMTs go through preamplifiers which have their gains adjusted so as to produce pulses with exactly the same height for monoenergetic gamma rays hitting a position on the detector corresponding to the center of each PMT.

The background correction procedure is carried out in two steps: first, a standard multiplicative flat-fielding procedure corrects for the apparent quantum efficiency variation across the detector; then the subtractive mask-antimask technique is applied to eliminate the systematic positiondependent background variations across the detector surface. . $^{29,30,33}$

The images presented here were obtained using a 320 $\mathrm{mCi}{ }^{137} \mathrm{Cs}$ radioactive source placed (aligned with a laser beam) in the center of the FOV, $68 \mathrm{~m}$ away from the PSD. This distance is long enough so as to make the magnification of the mask shadow negligible (the detector scale adjustment that would be needed, of $0.3 \mathrm{~mm}$, is much less than the detector spatial resolution of $\sim 1 \mathrm{~cm}$ ). The mask was placed $3.05 \mathrm{~m}$ away from the PSD, as it is in the final telescope configuration, and its rotation center was aligned with the geometrical center of the PSD. The image used for the flatfielding procedure was taken with the ${ }^{137} \mathrm{Cs}$ source placed at $\sim 20 \mathrm{~m}$ from the PSD without the mask (in order to obtain a uniform exposure) and for a long integration time (16 h).

In Fig. 6 we show a flat-fielded shadowgram cast on the detector surface by the ${ }^{137} \mathrm{Cs}$ source (plus background) in a 2 $\mathrm{h}$ integration in the above configuration (we selected the events inside the $662 \mathrm{keV}$ photopeak). The area shown corresponds to the complete central MURA pattern of the mask $(23.75 \mathrm{~cm} \times 23.75 \mathrm{~cm})$. One can clearly see the spatial distribution of the events according to the mask pattern as well as the progressive radial degradation of the spatial resolution towards the corners of the distribution. 


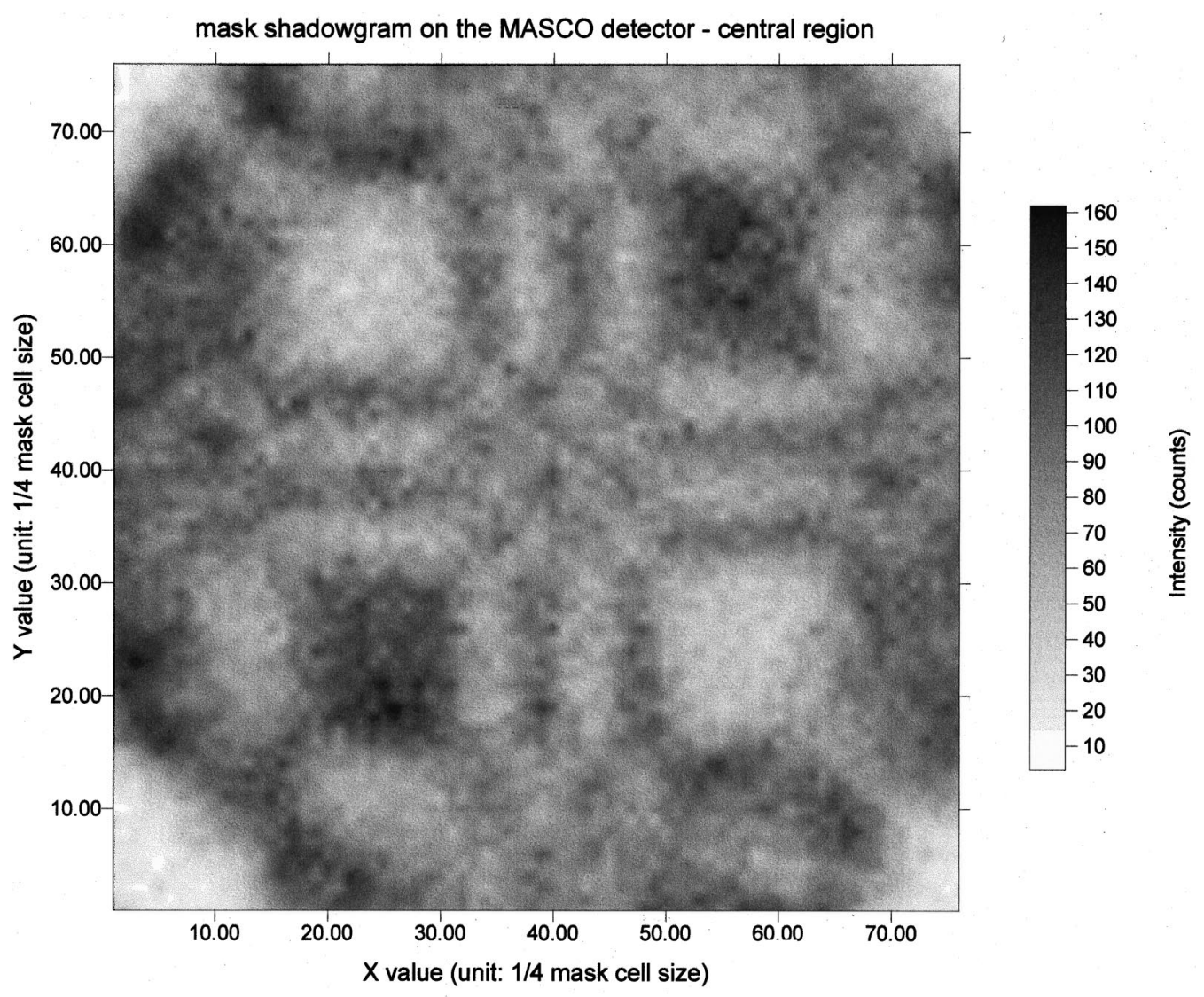

FIG. 6. Flat-fielded count distribution of $662 \mathrm{keV}$ photons coming from a ${ }^{137} \mathrm{Cs}$ source.

In Fig. 7 we present the reconstructed image corresponding to the count distribution of Fig. 6. The image was built using the cross-correlation method with the $G$ decoding function replaced by an array that represents the convolution of the extended original $G$ MURA pattern with the detector point-spread function (PSF) at $662 \mathrm{keV}^{22}$ Due to the effects caused by the radial degradation of the detector ability to determine correct positions (particularly at the corners of the central detector region used which corresponds to a complete MURA pattern) and background nonuniformity, the rms variation of the off-peak region of the image is a factor 7.5 higher than the Poisson value for the total number of counts, which means that the noise in the image is highly dominated by systematic effects rather than by statistical (Poisson) fluctuations. The signal-to-noise ratio (SNR) of this image is 14.8, whereas the purely Poissonian value would be $\sim 110$.

Finally, in Fig. 8 we present an image built using the subtractive mask-antimask technique, which was produced by correlating the PSF-convolved $G$ function of the mask with the difference between the recorded PSD counts with mask and antimask. The mask and the antimask recorded PSD counts were obtained with an integration time of $1 \mathrm{~h}$ each so that the statistics of this image are equivalent to that present in Fig. 7. The SNR of this image is of 23.7, a $60 \%$ improvement over the image of Fig. 7. This shows clearly the efficiency of the mask-antimask imaging technique when we have systematic variations in the background measured over the detector surface.

In order to get the purely Poissonian value for the SNR, one would have to use a detector with perfect spatial resolution and perfect ability to determine positions. With a nominal spatial resolution of about $1 \mathrm{~cm}$ full width at half maximum (FWHM) at $662 \mathrm{keV}$ (in the central $12 \mathrm{~cm}$ radius portion of the detector surface), which corresponds to $80 \%$ of the mask cell size, a loss of 35\% in SNR in comparison with the Poissonian value is expected based on empirical and simulation results. ${ }^{34,35}$ This means that the expected SNR at $662 \mathrm{keV}$ would by 71.5 and that our current best value of 23.7 is only $33 \%$ of what we expect to achieve. Since the mask-antimask technique does not introduce any intrinsic noise, as pointed out in Sec. III, we hope to improve the SNR of the images by reducing the noise of the data acquisition electronics, which in turn will improve the detector spatial resolution. A new low-noise data acquisition system is now under development. A major limitation, however, is the loss of position sensitivity at large radii, as explained above. As one can clearly see in Fig. 6, the positions at the corners of the central MURA region on the detector surface, used in the correlation analysis, are severely degraded. We are presently working on improvements of the position determination algorithm that hopefully are going to increase the maximum radius at which the detector can measure positions with reasonable precision.

\section{Mask rotation and source position ambiguity}

We produced a set of images of a $\sim 100 \mathrm{mCi}{ }^{237} \mathrm{Am}$ radioactive source, located $31 \mathrm{~m}$ away from the PSD, to test the rotating mask approach that eliminates the ambiguity in- 


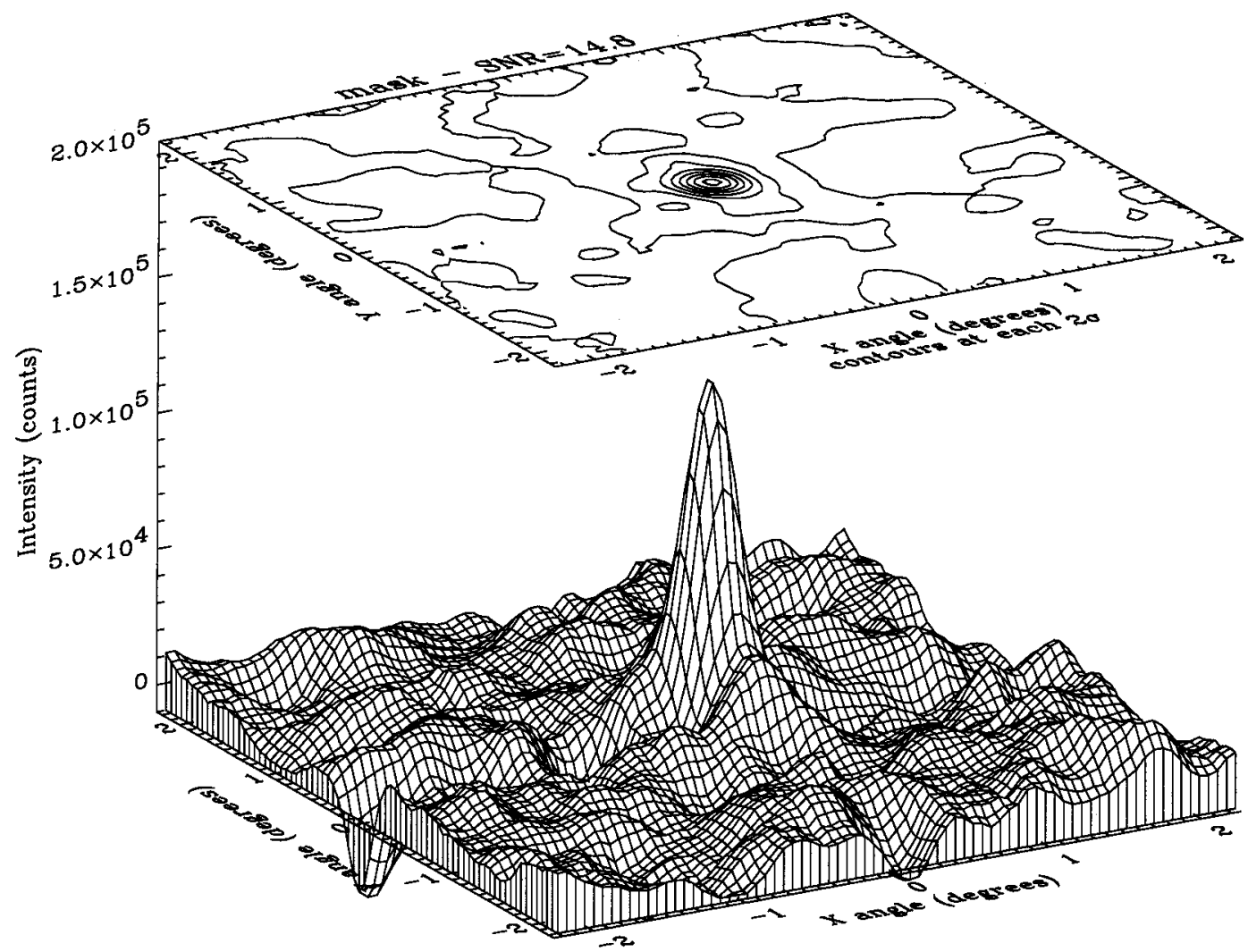

FIG. 7. Reconstructed image of a ${ }^{137} \mathrm{Cs}$ source placed in the center of the telescope's field of view. This image is not corrected for background nonuniformity. The signal-to-noise ratio is 14.8 .

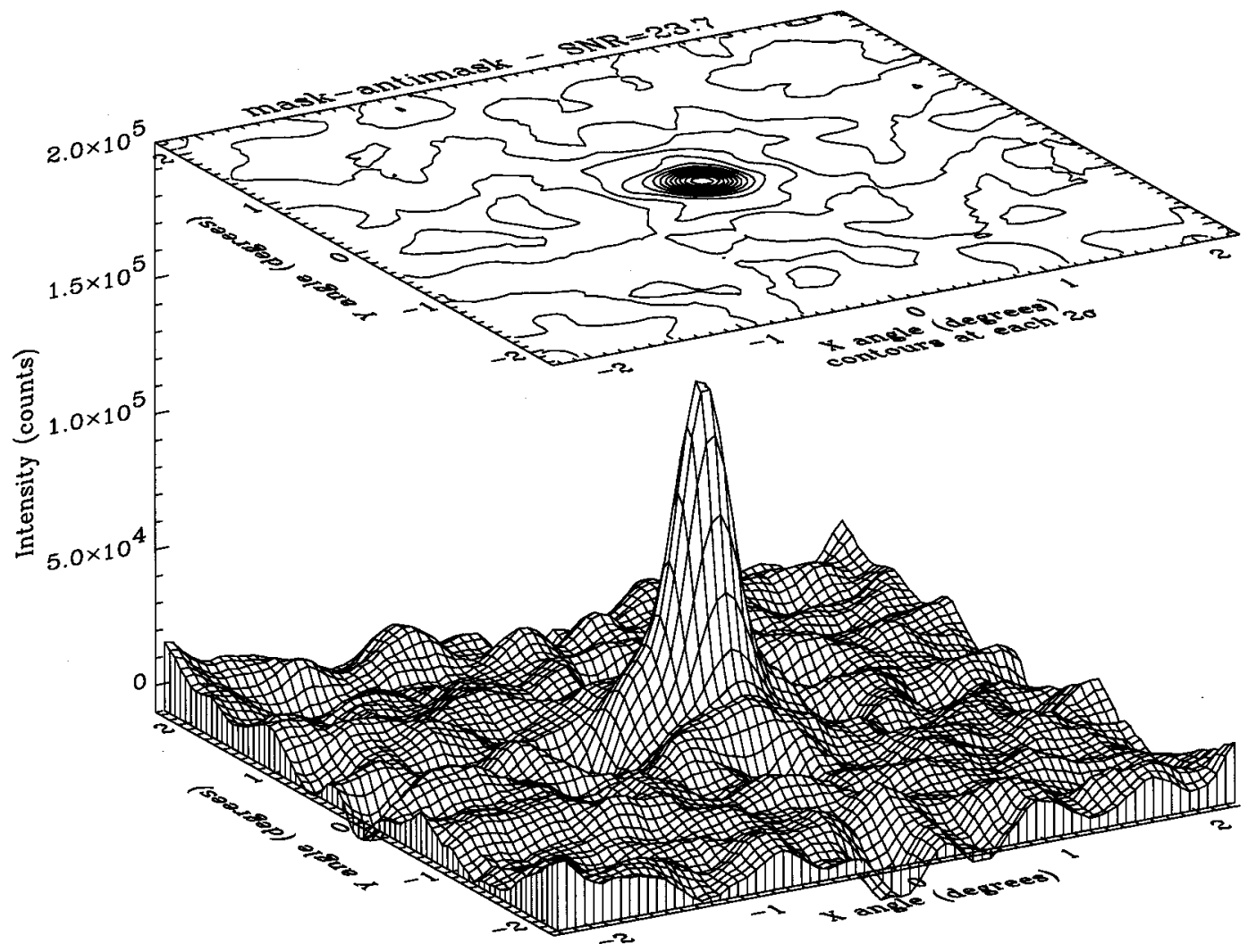

FIG. 8. Reconstructed image of a ${ }^{137} \mathrm{Cs}$ source placed in the center of the telescope's field of view. This image was obtained in the same laboratory setup as the one in Fig. 7, except that during half the integration, the mask was rotated to the antimask position. The signal-to-noise ratio is 23.7. 


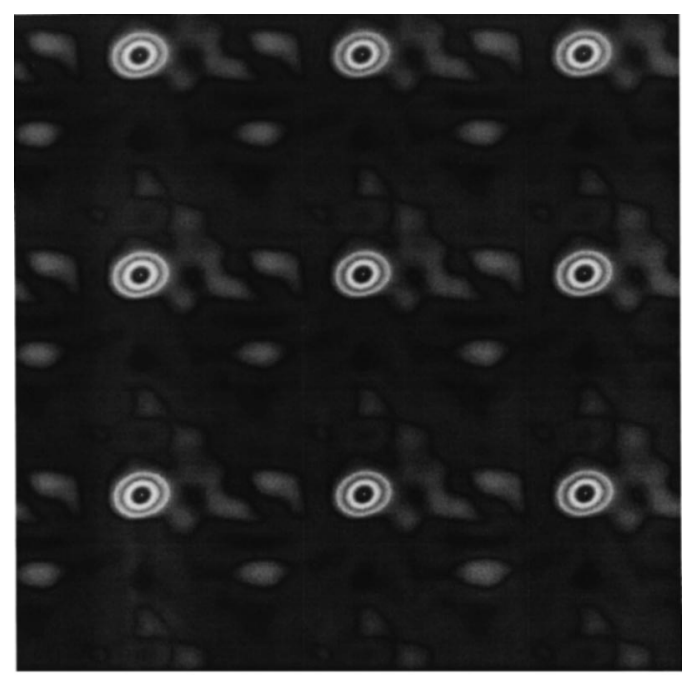

FIG. 9. Reconstructed image of the ${ }^{237} \mathrm{Am}$ radioactive source placed in the FCFOV, using the full extension of the mask. The SNR of all the nine peaks is $12.1 \pm 1.0$

troduced by the repetition of the basic MURA pattern. In this case we performed a scale adjustment on the PSD to account for the magnification of the shadowgram due to the finite distance to the source, even though, at this distance, the corrections in the event positions are still smaller than the detector spatial resolution. The results are shown in Figs. 9-11. In Fig. 9 we present a reconstructed image of the radioactive source placed in the FCFOV. This image was obtained using the mask-antimask technique and the complete extended mask pattern was used in the reconstruction procedure. The SNR of this image is of $12.1 \pm 1.0$. The presence of the nine possible positions of the source in the FOV, all of them producing the same shadow on the detector, is evident. In Fig. 10 , we show an image with the source in the same position and using the same integration time, but in this case the mask was continuously rotating. In this case, the true source position is evident and a set of rings around it is apparent. Those

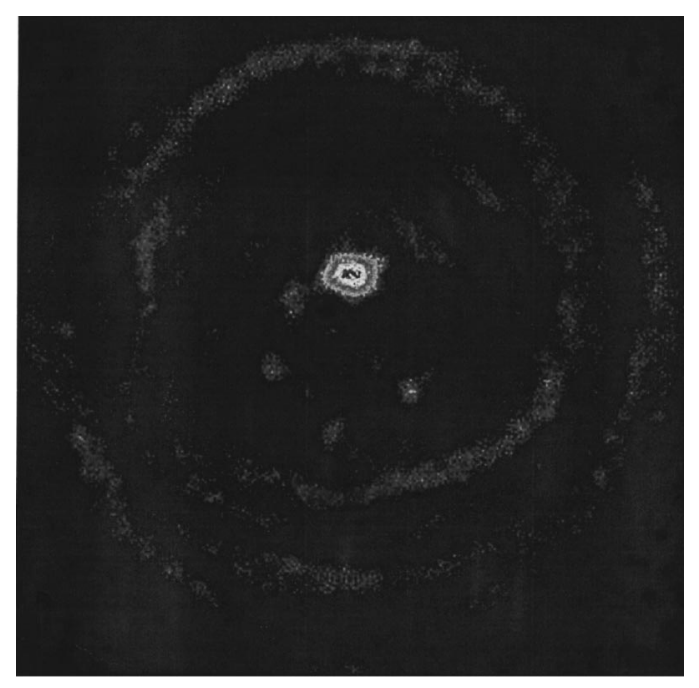

FIG. 10. An image with the source in the same position as in Fig. 9, for the same integration time, but in this case the mask is continuously rotating. The SNR is $14.3 \pm 1.0$.

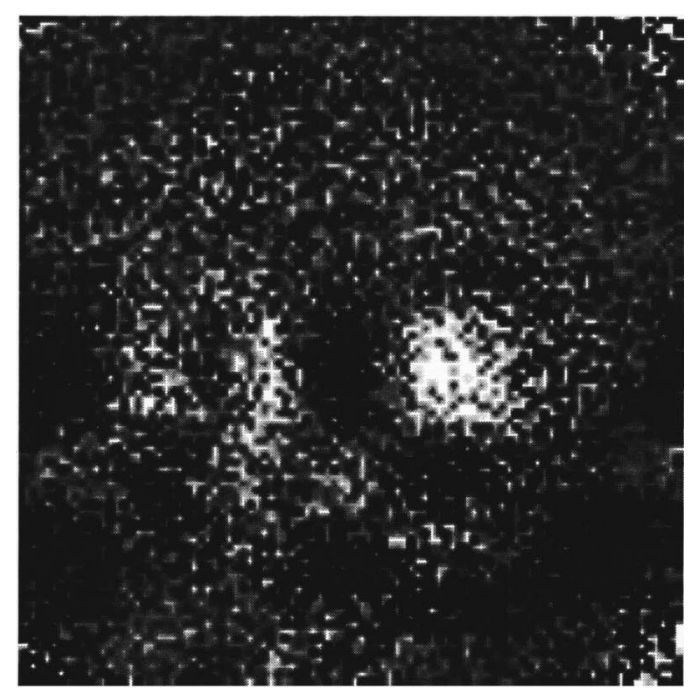

(a)

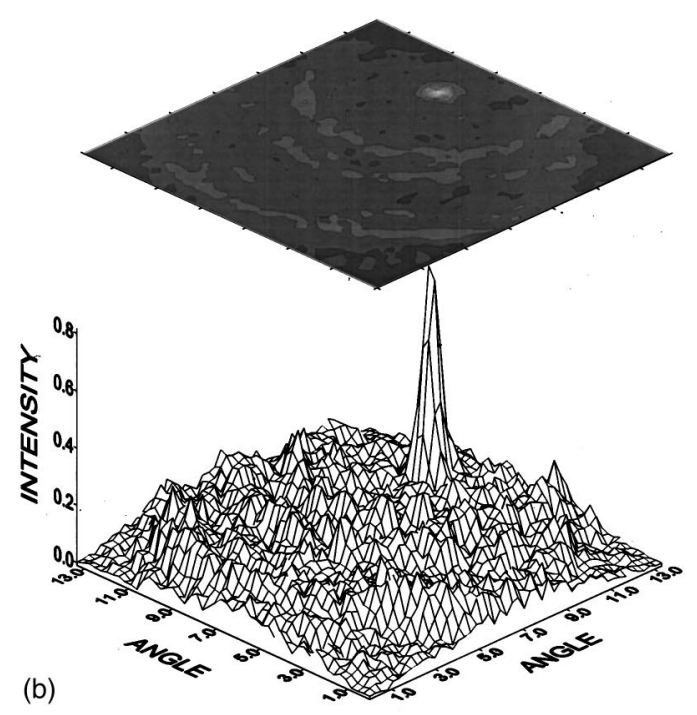

FIG. 11. (a) Shadowgram cast by the mask as registered by the detector system for a source located in the PCFOV, $8.3^{\circ}$ away from the telescope axis; (b) reconstructed image for the distribution in (a). The SNR is 8.7 \pm 1.0 .

rings are produced by the smearing of the "ghost" peaks. The SNR of this image (14.3 \pm 1.0$)$ is of the same order as the previous value, which indicates that the mask rotation does not introduce a loss in sensitivity. Finally, an image was obtained with the radioactive source in the PCFOV, $8.3^{\circ}$ away from the axis of the telescope. In Fig. 11(a) we show the shadow of the mask as registered by the detector system. Since the signal from the source does not cast a complete MURA shadow onto the detector, it introduces artifacts in the image, further degrading the SNR (which is already smaller than it should be in the case of the source in the PCFOV due to the loss of photons in the shields). Any source in the FCFOV also contributes to the background of the image as a whole, since a fraction of the photons will hit the detector surface without being coded by the mask. The fraction of this contribution is dependent on the position of the source in the PCFOV. In the Fig. 11(b) the reconstructed image of the source in the PCFV is shown. In this case, a SNR of $8.7 \pm 1.0$ was obtained. This is reasonable since, in 


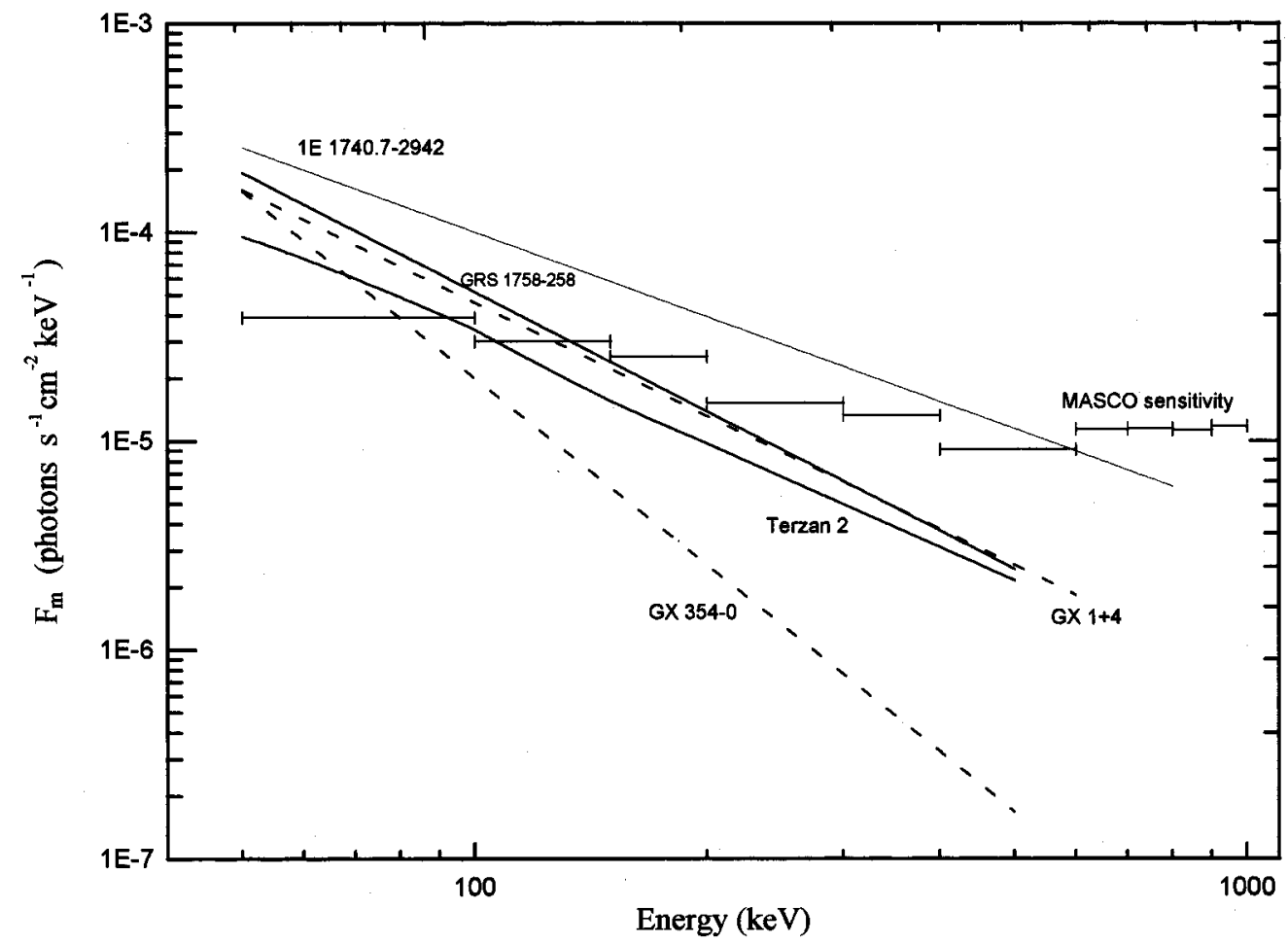

FIG. 12. $3 \sigma$ sensitivity of the MASCO telescope for an integration of $6 \mathrm{~h}$ at a residual atmosphere of $3.5 \mathrm{~g} / \mathrm{cm}^{2}$.

this case, approximately half of the photons from the source were coded by the mask.

\section{SENSITIVITY AND SIMULATED IMAGES}

The sensitivity of a conventional gamma-ray telescope, usually given in terms of the minimum detectable flux for a given statistical significance, is inversely proportional to its effective area and directly proportional to the square root of both the instrumental background counting rate and the time spent on both source and background integrations. ${ }^{34}$ On coded-aperture telescopes using (M)URA masks the sensitivity is essentially the same as if it were a conventional collimated instrument: whereas half the effective area is lost due to the mask, which would degrade the sensitivity by $\sqrt{2}$, source and background are measured simultaneously so that the factor of $\sqrt{2}$ is recovered. However, the detector spatial resolution plays an important role on these instruments, since the signal-to-noise ratio of the source detections on the reconstructed images depends on how sharp the mask shadowgram is defined in the PSD. ${ }^{34,35}$ For the MASCO telescope, we have chosen a mask cell size comparable to the detector spatial resolution (FWHM) so as to retain much of the angular resolution without sacrificing too much of the sensitivity. Furthermore, the signal-to-noise ratio for a point source in a coded-mask reconstructed image (when the aperture decoding function is unimodular) is given by the ratio of the net source counts by the square root of the net source counts plus the total number of counts in the detector. ${ }^{17}$ This should be taken into account when calculating the fluxes of the sources from coded-aperture reconstructed images. In Fig. 12 we show the sensitivity of the telescope together with the aver- age spectra of several Galactic Center (GC) x-ray sources (the $\mathrm{GC}$ region will be the main target of MASCO in its first balloon flight).

The background at balloon altitudes was estimated by direct scaling with the background measured by the Caltech's GRIP telescope over Fort Summer, NM. ${ }^{36}$ The counting rates were corrected for the geomagnetic rigidity of the Brazilian launching site $(11.7 \mathrm{GV})$. With the threshold of the anticoincidence system at $\sim 60 \mathrm{keV}$, the expected total counting rate from $50 \mathrm{keV}$ to $1.0 \mathrm{MeV}$ is of $\sim 230$ counts/s.

In order to demonstrate the point source sensitivity of the MASCO telescope, we performed a series of Monte Carlo simulations of the Galactic Center region. We have used the average fluxes of the main GC sources as measured by several instruments over the past few years. In this set of simulations, we have considered the fully coded square field of view determined by the central projection of the MURA pattern over the detector surface and we did not include marginal sources, since for this particular field we do not expect the presence of strong sources in the partially coded field of view. The simulated count distribution over the detector plane was produced by adding the shadowgrams of each source over the estimated background level. The source fluxes were calculated by integrating each spectrum over the image energy range and then correcting for MASCO's effective area and the integration time of the simulated observation. The positions of the detector hits, for each source, were produced by taking into account the incidence direction of each source. The positions were also Gaussian shifted according to the detector position resolution at each energy. This has the effect of smearing out the mask shadowgram cast on the detector. The final count distributions were then cross correlated with the mask pattern convolved with the 


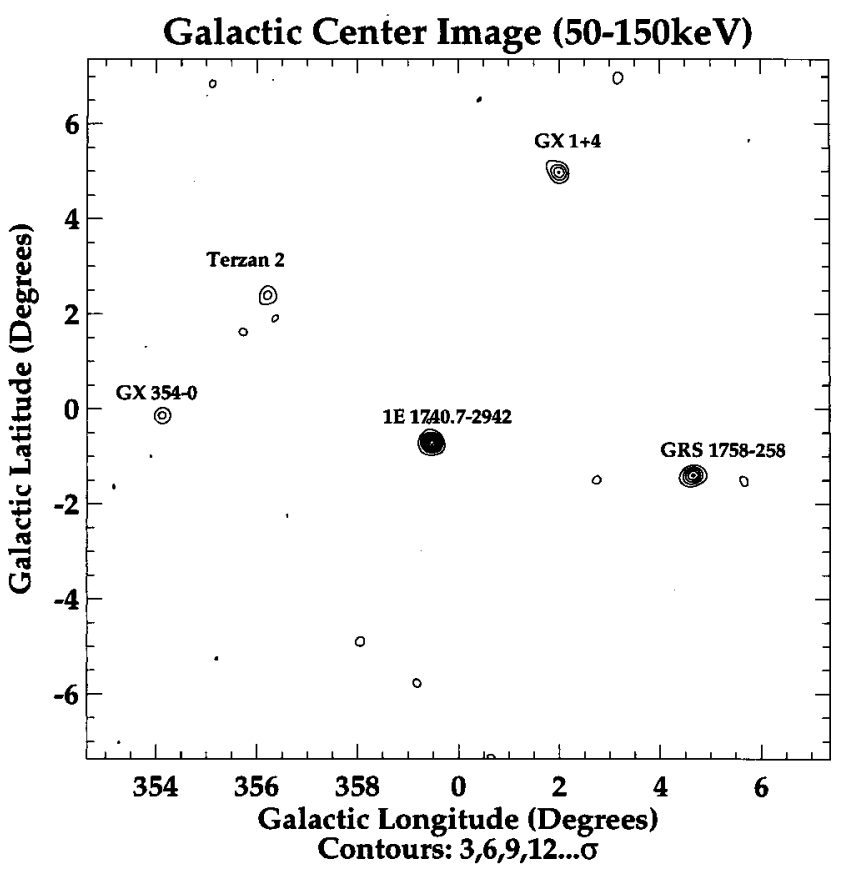

FIG. 13. Simulated image of the Galactic Center region by the MASCO telescope. The spectra of the GC sources were taken from the following references: 1E1740.7-2942 (see Ref. 37), GRS1758-258 (see Ref. 38), GX1+4 (see Ref. 39), GX354-0 (see Ref. 40), and Terzan 2 (see Ref. 41).

detector spatial resolution to produce the final images. In Fig. 13, a simulated image of the Galactic Center region is shown as it would be observed by a $6 \mathrm{~h}$ integration by the telescope at an atmospheric depth of $3.5 \mathrm{~g} / \mathrm{cm}^{-2}$ in the southeast part of Brazil.

\section{CONCLUSION}

The imaging system of MASCO balloon-borne codedaperture telescope has been tested in the laboratory and has shown interesting results, especially concerning the suppression of background position-dependent systematic effects over the detector plane. We devised a very simple and efficient way of implementing an antimask by using a $90^{\circ}$ antisymmetrical MURA pattern. The use of a mask-antimask technique using a MURA avoids the complex reconstruction algorithms involved in the use of hexagonal mask patterns. The application of this technique on laboratory images using a ${ }^{137} \mathrm{Cs}$ radioactive source provided a $\sim 60 \%$ increase in the sensitivity for intense point source detections in which the noise is highly dominated by systematic effects. The experiment has high-angular resolution $(\sim 14$ arcmin with a 4.5 arcmin location power for a $5 \sigma$ point source) and is very suitable for observations of crowded fields like the Galactic Center. It is hoped that the experiment will be launched onboard a stratospheric balloon by the end of the year 2002, in the Southern Hemisphere, with the main target being the Galactic Center region.

\section{ACKNOWLEDGMENTS}

This work was supported by FAPESP (Brazil) Grant Nos. 96/7638-3 and 93/2996-0, and CNPq (Brazil) Grants 302266/88-7/FA, 520677/96-0 and 466184100-0. J. M. was supported by CAPES. The authors thank L. A. Reitano and L. Arantes for invaluable work during the development of the mechanical implementation of the project. A. Alves, J. O. Fernandes, E. da Silva, L. de Almeida, and R. Correa are acknowledged for important contributions to the electronics design and mounting.

${ }^{1}$ H. R. Dicke, Astrophys. J. 153, L101 (1968).

${ }^{2}$ J. G. Ables, Proc. Astron. Soc. Aust. 4, 172 (1968).

${ }^{3}$ A. Willmore, G. Skinner, C. Eyled, and B. Ramsey, Space Sci. Rev. 30, 601 (1981).

${ }^{4}$ R. A. Sunyaev, S. I. Babichenko, D. A. Goganov, S. R. Tabaldyev, and N. S. Jambourenko, Adv. Space Res. 10, 233 (1990).

${ }^{5}$ R. Jager, W. A. Mels, A. C. Brinkman, M. Y. Galama, H. Goulooze, J. Heise, P. Lows, J. M. Muller, A. Naber, A. Rook, R. Schuurhof, J. J. Schuurmans, and G. Wiersma, Astron. Astrophys., Suppl. Ser. 125, 557 (1997).

${ }^{6}$ A. M. Levine, H. Bradt, W. Cui, J. G. Jernigan, E. H. Morgan, H. Edward, R. Remillard, R. E. Shirey, and D. A. Smith, Astrophys. J. Lett. 469, L33 (1996).

${ }^{7}$ S. M. Schindler, W. R. Cook, J. Hammond, F. A. Harrison, T. A. Prince, S. Wang, S. Corbel, and W. A. Heindl, Nucl. Instrum. Methods 384, 425 (1997).

${ }^{8}$ M. Garcia, J. Grindlay, R. Burg, S. Murray, and J. Flanagan, IEEE Trans. Nucl. Sci. 33, 735 (1986).

${ }^{9}$ J. Braga, C. Covault, and J. E. Grindlay, IEEE Trans. Nucl. Sci. 36, 871 (1989).

${ }^{10}$ J. Roques, J. Paul, P. Mandrou, and F. Lebrun, Adv. Space Res. 10, 223 (1990).

${ }^{11}$ J. Paul, J. Ballet, M. Cantin, B. Cordier, A. Goldwurm, A. Lambert, P. Mandrou, J. P. Chabaud, M. Ehanno, and J. Lande, Adv. Space Res. 11, 289 (1991).

${ }^{12}$ R. Sunyaev, E. Churazov, M. Gilfanov, M. Pavlinsky, S. Grebenev, G. Babalyan, I. Dekhanov, N. Yamburenko, L. Bouchet, M. Neil, J.-P. Roques, P. Mandrou, A. Goldwurm, B. Cordier, Ph. Laurent, and J. Paul, Astron. Astrophys. 247, L29 (1991).

${ }^{13}$ L. Bouchet, J. P. Roques, J. Ballet, A. Goldwurm, and J. Paul, Astrophys. J. 548, 990 (2001).

${ }^{14}$ A. Goldwurm, M. Denis, J. Paul, S. Faisse, J.-P. Roques, L. Bouchet, G. Vedrenne, P. Mandrou, R. Sunyaev, E. Churasov, M. Gilfanov, A. Finogenov, A. Vikhlinin, A. Dyachkov, N. Khavenson, and V. Kovtunenko, Adv. Space Res. 15, 41 (1995).

${ }^{15}$ A. Goldwurm, B. Cordier, J. Paul, J. Ballet, L. Bouchet, J.-P. Roques, G. Vedrenne, P. Mandrou, R. Sunyaev, E. Churazov, M. Gilfanov, A. Finogenov, A. Vikhlinin, A. Dyachkov, N. Khavenson, and V. Kovtunenko, Nature (London) 371, 589 (1994).

${ }^{16}$ M. Vargas, A. Goldwurm, J. Paul, M. Denis, V. Borrel, L. Bouchet, J.-P. Roques, E. Jourdain, S. Trudolyubov, M. Gilfanov, E. Churazov, R. Sunyaev, N. Khavenson, A. Dyachkov, B. Novikov, and I. Chulkov, Astron. Astrophys. 313, 828 (1996).

${ }^{17}$ E. E. Fenimore and T. M. Cannon, Appl. Opt. 17, 337 (1978).

${ }^{18}$ S. R. Gottesman and E. E. Fenimore, Appl. Opt. 28, 4344 (1989).

${ }^{19}$ T. Villela, J. Braga, F. D'Amico, and J. A. Neri, Astrophys. Space Sci. 214, 161 (1994)

${ }^{20}$ T. Villela, J. Braga, F. D'Amico, and U. B. Jayanthi, Adv. Space Res. 15, 95 (1995).

${ }^{21}$ F. D'Amico, M. M. Hamada, C. H. Mesquita, J. M. Vieira, N. P. Costa Júnior, T. Villela, and J. Braga, Nucl. Instrum. Methods Phys. Res. A 422, 139 (1999).

${ }^{22}$ W. E. Althouse, W. R. Cook, A. C. Cummings, M. H. Finger, T. A. Prince, S. M. Schindler, C. H. Starr, and E. C. Stone, Proceedings of the 19th International Cosmic Ray Conference, La Jolla, CA, 1985, Vol. 3, p. 299.

${ }^{23} \mathrm{~J}$. Mejía, Ph.D. thesis, Instituto Nacional de Pesquisas Espaciais (INPE), São José dos Campos, SP, Brazil, 2002.

${ }^{24}$ T. Villela, R. A. Fonseca, P. de Souza, A. Alves, J. Mejía, R. Corrêa, and J. Braga, Adv. Space Res. 26, 1415 (2000).

${ }^{25}$ J. Mejía, T. Villela, and J. Braga, Adv. Space Res. 26, 1407 (2000).

${ }^{26}$ K. Byard, Exp. Astron. 2, 227 (1992).

${ }^{27}$ F. D'Amico, Ph.D. thesis, Instituto Nacional de Pesquisas Espaciais (INPE), São José dos Campos, SP, Brazil, 1997.

${ }^{28}$ U. B. Jayanthi and J. Braga, Nucl. Instrum. Methods Phys. Res. A 310, 685 (1991). 
${ }^{29}$ J. Braga, T. Villela, U. B. Jayanthi, F. D'Amico, and J. A. Neri, Exp. Astron. 2, 101 (1991).

${ }^{30}$ J. Braga, F. D’Amico, and T. Villela, Exp. Astron. 5, 269 (1994).

${ }^{31}$ J. Braga, F. D'Amico, and T. Villela, Adv. Space Res. 15, 157 (1995).

${ }^{32}$ Manual 6406, H. P. Scintillation Camera and Tomo Accessory (NuclearChicago, 1971).

${ }^{33}$ F. D'Amico, J. Braga, T. Villela, A. M. R. Alves, E. R. Silva, and J. A. Neri, Rev. Fis. Apl. Instr. 10, 15 (1995).

${ }^{34}$ J. Braga, Ph.D. thesis, IAG, University of São Paulo, Brazil, 1990

${ }^{35}$ E. Caroli, J. B. Stephen, G. Di Cocco, L. Natalucci, and A. Spizzichino, Space Sci. Rev. 45, 349 (1987).

${ }^{36}$ M. H. Finger, Ph.D. thesis, California Institute of Technology, 1987.

${ }^{37}$ S. A. Grebenev, M. N. Pavlinsky, and R. A. Sunyaev, Adv. Space Res. 15, 115 (1995).
${ }^{38}$ S. Mereghetti, T. Belloni, and A. Goldwurm, Astrophys. J. Lett. 433, L21 (1994).

${ }^{39}$ S. W. B. Dieters, J. G. Greenhill, D. P. Sharma, R. K. Sood, L. Waldron, and M. C. Storey, Adv. Space Res. 11, 35 (1991).

${ }^{40}$ A. Claret, A. Goldwurm, B. Cordier, J. Paul, J.-P. Roques, L. Bouchet, M.-C. Schmitz-Fraysse, P. Mandrou, E. Churazov, M. Gifanov, R. Sunyaev, N. Khavenson, A. Diachkov, B. Novikov, R. Kremnev, and V. Kovtunenko, Astrophys. J. 423, 436 (1994).

${ }^{41}$ D. Barret, S. Mereghetti, J.-P. Roques, P. Mandrou, L. Salotti, F. Lebrun, $\mathrm{Ph}$. Laurent, J. Ballet, E. Churazov, M. Gilfanov, R. Sunyaev, N. Khavenson, I. Chulkov, B. Novikov, A. Kuznetzov, and A. Dyachkov, Astrophys. J. Lett. 379, L21 (1991). 